\title{
Design and modeling of reliable networks (foreword)
}

\author{
Jacek Rak • Brigitte Jaumard · Roland Wessäly
}

Published online: 30 August 2013

(c) The Author(s) 2013. This article is published with open access at Springerlink.com

This Special Issue presents extended versions of selected papers from 3rd International Workshop on Reliable Networks Design and Modeling (RNDM 2011) held in Budapest, Hungary on October 3-5, 2011. This event, organized by Gdansk University of Technology (PL) in co-operation with Concordia University (CA), and atesio $\mathrm{GmbH}(\mathrm{DE})$, was technically co-sponsored by IFIP TC6 WG 6.10 and IEEE.

Similar to previous editions, RNDM 2011 provided a very successful discussion forum for people from academia and industry. After a detailed review process, 27 accepted papers written by authors from 23 countries, were organized into seven technical sessions, namely: "Optical Networks Survivability", "Resilience of Multilayer and Overlay Networks", "Models and Algorithms of Survivable Networks Design", "Network Reliability Assessment", "Theory of Network Reliability", "Survivability of Anycast and Multicast Networks", and "Shared Link Risk Groups".

Authors of 15 papers of high merit were next invited to submit the extended versions to this Special Issue. Final acceptance of papers included here was conditional upon re-

\section{J. Rak $(\varangle)$}

Faculty of Electronics, Telecommunications and Informatics, Gdansk University of Technology, Narutowicza 11/12, 80-233, Gdansk, Poland

e-mail: jrak@pg.gda.pl

B. Jaumard

CSE-Computer Science and Software Engineering, Concordia University, 1455 De Maisonneuve Blvd. West, EV-003-189,

Montreal, Quebec, H3G 1M8, Canada

e-mail: bjaumard@cse.concordia.ca

R. Wessäly

atesio $\mathrm{GmbH}$, Bundesallee 89, 12161 Berlin, Germany

e-mail: wessaely@atesio.de ceiving appropriate positive review scores, and carefully addressing the reviewers' remarks. Each of the papers is briefly introduced below.

The paper Resilient Network Design: Challenges and Future Directions, by David Tipper provides an overview of current trends and directions in reliable networks design. In particular, it discusses the complexity and challenges of providing reliable services in evolving communications infrastructure. James P.G. Sterbenz et al. discuss in their paper entitled Redundancy, Diversity, and Connectivity to Achieve Multilevel Network Resilience, Survivability, and Disruption Tolerance three critical resilience disciplines and the corresponding mechanisms to achieve multilevel resilience, namely: redundancy for fault tolerance, diversity for survivability, and connectivity for disruption tolerance. The paper entitled Highly Available Network Design and Resource Management of SINET4 by Shigeo Urushidani et al. presents a very interesting analysis of a Japanese academic backbone network reliability following from real information achieved during the disastrous March 11 Great East Japan Earthquake.

In Path Diversification for Future Internet End-to-End Resilience and Survivability, Justin Rohrer et al. describe a new method called "path diversification" providing multiple paths to increase the reliability of flows. A $\mathrm{Hy}$ brid Rerouting Scheme by Akli Fundo et al. includes another important proposal of a method aimed to provide cost-efficient reliable routing by means of combining the following three restoration techniques, namely path diversity, end-to-end rerouting with stub release, and global rerouting. Investigations on all-terminal reliability are presented in a paper Inflection Points for Network Reliability by Jason Brown et al. In particular, the authors show that the all-terminal reliability of almost every simple graph has a point of inflection. Another important result is that there are 
infinite families of graphs with more than one point of inflection.

The next two papers refer to the concept of loop-free alternate path (LFA). In particular, Wouter Tavernier et al. in Self-configuring Loop-Free Alternates with High Link Failure Coverage present a logical extension of the loop-free alternate concept, introducing a self-configuring scheme to populate the corresponding alternate entries, and provide the respective evaluation of the method performance in terms of obtained coverage, configuration time, and path length. In Optimization Methods for Improving IP-level Fast Protection for Local Shared Risk Groups with Loop-Free Alternates, Máté Nagy et al. include a novel bipartite graph model for the LFA graph problem extended to the case of multiple failures using the concept of Shared Risk Groups, and present a set of heuristic algorithms to obtain the approximate solutions.

The next paper by Michał Kucharzak and Krzysztof Walkowiak entitled Modeling and Optimization of Maximum Flow Survivable Overlay Multicast with Predefined Routing Trees refers to multicast traffic protection in overlay networks aimed at realizing maximum throughput with survivability constraints.

Providing protection of flows by means of protection cycles ( $p$-cycles) is another recent topic addressed in papers A Heuristic Approach to Working and Spare Capacity Optimization for Survivable Anycast Streaming Protected by $p$-Cycles by Adam Smutnicki and Krzysztof Walkowiak, and Robust FIPP p-Cycles against Dual Link Failures by Brigitte Jaumard et al. The former one provides solutions for anycast traffic protection, while the latter paper presents the application of Failure-Independent Path-Protecting (FIPP) $p$-cycles to achieve protection against simultaneous failure of two links.

Next three papers address the issue of optical networks reliability. The first of them entitled Survey on Out-of-Band Failure Localization in All-Optical Mesh Networks by Janos Tapolcai presents an overview of recent research trends with respect to out-of-band monitoring. The paper Methods for Physical Impairment Constrained Routing with Selected Protection in All-Optical Networks by Peter Soproni et al. analyzes the problem of survivable alloptical routing in WDM networks with physical impairments. In particular, the authors introduce methods to maximize the number of demands routed with protection in alloptical way in capacity-constrained networks with limitations on path lengths according to physical impairments. Ali Shaikh and Brigitte Jaumard introduce in Optimized Dimensioning of Resilient Optical Grids with Respect to Grade of Services a scalable optimization model for maximizing IT services in optical grids subject to survivability mechanisms.

The last paper by Mateusz Żotkiewicz and Michał Pióro entitled Exact Approach to Reliability of Wireless Mesh Net- works with Directional Antennas refers to reliability of wireless mesh networks-being currently an emerging area of research. In particular, the authors discuss an optimal way of solving the cost minimization problem. An extensive numerical study is presented to illustrate the efficiency of the proposed method.

The editors of this Special Issue would like to express their gratitude to the Editor of TSJ for his consent to publish RNDM 2011 materials in the Journal, as well as to reviewers for delivering the detailed reviews.

Open Access This article is distributed under the terms of the Creative Commons Attribution License which permits any use, distribution, and reproduction in any medium, provided the original author(s) and the source are credited.

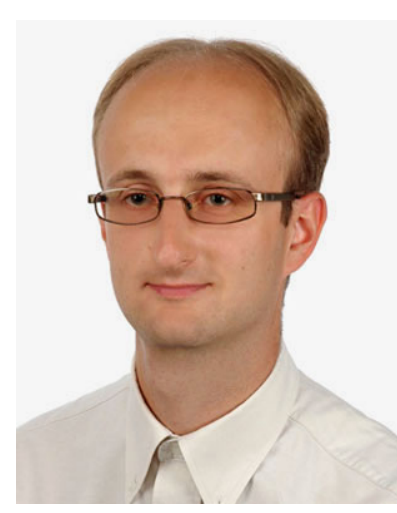

Jacek Rak holds Ph.D. degree in computer science (option: computer communications), received with distinction in 2009 from Gdansk University of Technology (GUT), Poland. He is currently an assistant professor at the Department of Computer Communications of the Faculty of Electronics, Telecommunications and Informatics at GUT. His main research areas include: routing, design, dimensioning and analysis of high-speed networks with focus on survivability.

Dr. Rak was involved in many projects related to optimization of reliable computer networks. He is the founder and General Chair of International Workshop on Reliable Networks Design and Modeling (RNDM), Steering Committee Member of ICUMT, and RNDM. He served as a TPC Co-Chair of Networks 2010, ICUMT 2011, Publication Chair of BCFIC 2011\&2012, NETWORKS 2012, TPC Member of major conferences in communications, as well as a reviewer of journals including e.g., IEEE/ACM Transactions on Networking, IEEE Communication Letters, Computer Communications (Elsevier). He is also a member of IEEE, and a Co-chair of IFIP TC6 WG 6.10 (Photonic Networking Group).

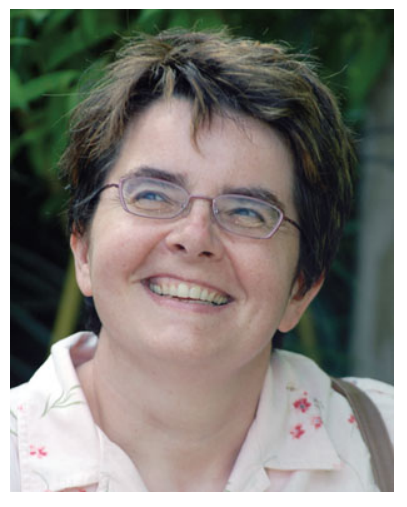

Brigitte Jaumard holds a Concordia University Research Chair, Tier 1, on the Optimization of Communication Networks in the Computer Science and Software Engineering Department at Concordia University. Her research focuses on mathematical modeling and algorithm design for large-scale optimization problems in communication networks and artificial intelligence. Recent studies include the design of efficient algorithms for $p$-cycle based protection schemes, under static and dynamic traffic, and their generalization to the so-called $p$-structures. Other recent studies deal with provisioning and scheduling algorithms in optical grids, in broadband wireless networks and in passive optical networks. In Artificial Intelligence, contributions include the development of efficient optimization algorithms for probabilistic logic (reasoning under uncer- 
tainty) and for automated mechanical design in social networks (design of trust estimator tools). B. Jaumard has published over 200 papers in international journals in Operations Research and in Telecommunications.

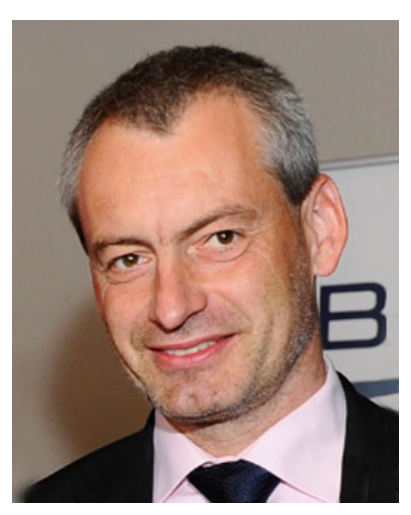

Roland Wessäly is a co-founder and managing director of atesio. His work at the Zuse-Institute Berlin, a world-wide leading research institute in discrete mathematics, resulted 2000 in his Ph.D. In 2001 he received the renowned Vodafone Innovations Award for fundamental contributions to the cost-optimal design of survivable communication networks. Under his direction, numerous sophisticated and efficient algorithms based on integer programming models for fixed network planning and optimization including SDH, IP over WDM, and FTTx network design and configuration have been developed, which are in daily use in the planning departments of large network operators. 